\title{
On a boom and bust year class cycle
}

\author{
ODO DIEKMANN* $*^{*}$, NATALIA DAVYDOVA $\dagger$ and STEPHAN VAN GILS $\ddagger$ \\ $\dagger$ Department of Mathematics, Utrecht University, Utrecht, the Netherlands \\ $\$$ Faculty of Mathematical Sciences, University of Twente, 7500AE Enschede, the Netherlands
}

(Received 13 August 2004; in final form 3 November 2004)

Dedicated to Jim Cushing for all the inspiration, ever since Cortona 1979.

\begin{abstract}
We motivate and describe a class of nonlinear Leslie matrix models for semelparous populations, like cicadas and Pacific salmon. We then focus on a Cushing-inspired special case for which one can show rigorously that a heteroclinic boundary cycle exists and attracts nearby orbits for a certain range of parameters. Along the way we formulate some open problems concerning a carrying simplex and global behaviour.
\end{abstract}

Keywords: Heteroclinic cycle; Leslie matrix model; Semelparous species

\section{What is a year class?}

A species is called semelparous if reproduction also amounts to signing one's own death sentence. When there is exactly one reproduction opportunity per year (usually in the spring), we take one year as a natural discrete time unit. For several species, in particular many cicada species, the period in between being born and going to reproduce is strictly fixed at, say, $k$ years. The population then subdivides into subpopulations according to the year of birth modulo $k$ (or, equivalently, the year of reproduction modulo $k$ ). Such a subpopulation is called a year class (or, also, a brood). Note that whereas the age class to which an individual belongs increases by one when a year has passed, the year class to which it belongs is fixed once and for all.

Year classes only mate among themselves and hence are reproductively isolated. In particular, if a year class goes extinct, it remains extinct. We then say that the year class is "missing". The periodical insects [1] are those for which all but one year classes are missing. The prime example is the Magicicadas (the $k=17$ species had its most recent emergence in the North-East United States in 2004).

\section{Competition among year classes}

Even though year classes are, by definition, reproductively isolated, they may still interact (either by direct competition for food or space, i.e. access to feeding spots, or by apparent

*Corresponding author. Email: diekmann@math.uu.nl

Journal of Difference Equations and Applications

ISSN 1023-6198 print/ISSN 1563-5120 online (C) 2005 Taylor \& Francis Group Ltd

http://www.tandf.co.uk/journals

DOI: $10.1080 / 10236190412331335409$ 
competition via cannibalism, predators or parasites). Often it is not at all clear how exactly the interaction works in case of a particular species such as e.g., the Magicicadas. It is therefore of interest to classify mechanisms of interaction according to whether or not they lead, when incorporated in a mathematical model of the population dynamics, to competitive exclusion of $k-1$ year classes. More generally one can ask how many year classes are expected to coexist (and how the answer depends on the specific assumptions underlying the model)?

A particular feature of year classes is that there are no intrinsic differences whatsoever among the individuals comprising different year classes. This feature is rather non-generic in the context of general competition models, but part of the definition in the present case. The emerging difference, however, derives from the timing of birth, which determines how the phase within the life cycle compares to the phase in the cycle of environmental conditions generated by the population dynamics itself. Here the "environmental conditions" should capture everything which has an influence on survival and reproduction (as well as maturation whenever this is, unlike the case of age structure, proceeding at a variable rate) and which, in turn, is influenced by the population size and composition. In other words, all interactions between individuals is via a feedback loop involving variable environmental conditions (cf. [8,9]).

The key questions then are: can one year class set the environmental conditions (as a function of time) such that all other year classes, when rare, are driven to extinction? Or are missing year classes able to invade successfully? Do we get coexistence or competitive exclusion? Or are there still other possibilities?

\section{Nonlinear Leslie matrix models}

To enhance the readability, we formulate the model for the case $k=3$, trusting that it is clear from this representative case how to generalize to arbitrary values of $k$. Let $N_{i}(t)$ denote the density of the $i$-th age class in year $t$, where $i=0,1,2$ (following the tradition in some biological literature, we number the lowest age class 0 : if the census moment is shortly after the birth event, this is indeed quite appropriate). We refer to [3] for a general introduction to nonlinear Leslie matrix models. Let

$$
L(h)=\left(\begin{array}{ccc}
0 & 0 & h_{2} \\
h_{0} & 0 & 0 \\
0 & h_{1} & 0
\end{array}\right)
$$

denote the (irreducible but non-primitive) Leslie matrix with survival probabilities $h_{0}, h_{1}$ and combined survival-reproduction factor $h_{2}$. The product of three such matrices is, irrespective of whether or not we take the same vector $h$, a diagonal matrix. This is the mathematical fact that corresponds to the possibility of year classes being missing. Indeed, it leads to invariance of coordinate planes and axes under the $k$ times iterated map, which we shall call the full-life-cycle map.

We do not consider $h$ as constant but rather consider $h=h(I(t))$ where $I(t)$ represents the environmental condition at time $t$.

A detailed description of interaction would take the form of a continuous time model for the period in between two birth events. For instance, if $S$ denotes substrate (= food) and $c_{i}$, 
with $c_{0}+c_{1}+c_{2}=1$, are the relative feeding rates of the age classes, we could postulate

$$
\frac{\mathrm{d} S}{\mathrm{~d} \tau}=K-\alpha\left(c_{0} N_{0}+c_{1} N_{1}+c_{2} N_{2}\right) S,
$$

where $\tau$ with $0 \leq \tau \leq 1$ denotes "time within the year", $K$ is the constant rate at which substrate is produced and $\alpha$ is the "absolute" feeding rate. If both $K$ and $\alpha$ are large we can make the quasi-steady-state assumption

$$
S=\frac{K}{\alpha\left(c_{0} N_{0}+c_{1} N_{1}+c_{2} N_{2}\right)}
$$

We then define

$$
I=c_{0} N_{0}+c_{1} N_{1}+c_{2} N_{2}
$$

and let $h$ be the composite function that incorporates how survival and reproduction depend on $S$ and how $S$ depends on $I$ (viz., as $S=K / \alpha I$ ). The weak point in this story is that we have separated feeding, as described by equation (3.2), from survival, as incorporated in $h$ (Indeed, we took the $N_{i}$ in equation (3.2) as constants and did not incorporate the reduction of age class size by death). Nevertheless we take equation (3.4) as the defining feedback relation between $I$ and the current population size and composition, while admitting that this makes the model a bit phenomenological (such in contrast to a model that is mechanistic in every detail).

We assume that $h_{i}$ is a decreasing function of $I$ for $i=0,1,2$. Even so we should distinguish between Beverton-Holt type cases, in which $I \mapsto I h_{i}(I)$ is increasing, and Ricker type cases, in which $I \mapsto I h_{i}(I)$ has a one-humped graph (i.e. it is unimodal).

The discrete time dynamical system that we shall study is generated by the recursion

$$
N(t+1)=L(h(I(t))) N(t) \quad I(t)=c_{0} N_{0}(t)+c_{1} N_{1}(t)+c_{2} N_{2}(t) .
$$

The basic question is: how does the attractor structure depend on the ingredients $h$ and $c$ ? In addition we like to know how the answer to this question depends on the value of $k$ ?

Our aim is to investigate the influence of the year class structure and not to study complex dynamics per se. Therefore we restrict our attention to Beverton-Holt type nonlinearities, for which the corresponding one dimensional map would show convergence of all orbits towards the positive steady state for all values of parameters for which the positive steady state exists (or equivalently, the basic reproduction ratio $R_{0}$ exceeds one).

\section{The dichotomy for $k=2$.}

In [6] we showed that for $k=2$ there is a strict dichotomy:

- either the coexistence steady state is (locally) stable,

-or the period two cycle on the axes (corresponding to a single year class being present) is (locally) stable.

The transition between these two cases is by way of a vertical period-doubling bifurcation. By this we mean the following. A stability switch of the coexistence steady state 
occurs by way of a multiplier passing the value -1 . For exactly the parameter values for which the multiplier equals -1 , there is a one-parameter family of period two orbits. This family connects the coexistence steady state to the two-cycle at the boundary (of the positive cone). Accordingly the boundary two-cycle undergoes a stability switch at exactly those same parameter values.

We can actually apply the results of [11], which are based on the order induced by the cone $K=\left\{\left(x_{0}, x_{1}\right): x_{0} \geq 0, x_{1} \leq 0\right\}$, to conclude that in the first case the coexistence steady state is a global attractor for the interior of the positive cone. Namely, we can show that the full-life-cycle map with the Beverton-Holt type nonlinearities is competitive in $\mathbb{R}_{2}^{+}$. Since in addition, this map is injective there and has a positive determinant, one can perform an analysis similar to one in [11, Proposition 6.3] to show that in the second case almost every orbit is asymptotic to the period-two orbit at the boundary, with the asymptotic phase determining which of the two year classes goes extinct (the exceptional orbits constitute the one-dimensional stable manifold of the coexistence steady state, which forms the separatrix for the two basins of the "phase" attraction). Besides we conjecture that there exists a unique and attractive carrying simplex (cf. [12]; one can think of it as the boundary of the domain of repulsion of the repellor at 0 ).

In fact, we believe that similar results can also be obtained in the case of non-monotone (Ricker-like) nonlinearities, if the values of the basic reproduction ratio $R_{0}$ are just above 1 . But we now turn to $k=3$.

\section{An additional possibility for $k=3$.}

For $k=3$ too one can have a locally stable coexistence steady state as well as, for different parameter values, three locally stable period 3 points corresponding to two year classes being missing (Remarkably, the situation in which only one year class is missing is never stable.) The vertical bifurcation in which a two-dimensional simplex is filled with period 3 orbits is now a co-dimension two phenomenon.

The "usual" way in which the coexistence steady state turns unstable is by way of a Neimark-Sacker bifurcation that yields an invariant circle.

But there is an additional possibility: the three single year class period 3 points on the axes can have connecting orbits, thus forming a rock-scissors-paper heteroclinic cycle at the boundary (cf. [10], Section 7.7), which can be an attractor (in fact its stability character can change when it absorbs or spits out an invariant circle). In such a cycle the year classes are chasing each other, one growing at the expense of the decline of its predecessor and so on and on eternally, with the periods of hegemony of a year class becoming longer and longer.

Precise statements and proofs of most statements above can be found in [7] or in [5], but concerning the existence of the heteroclinic boundary cycle there is only analytical and numerical evidence but not yet a complete proof (again it would be most helpful if we had results about a two-dimensional carrying simplex).

After reading [2] we realized that for a special case one can prove rather easily that a heteroclinic boundary cycle exists. The next section is devoted to (an analysis of) that special case. 


\section{A special case inspired by "Cycle chains and the LPA model"}

The key features of the special case are that $h_{0}$ and $h_{1}$ are constant while $c_{1}=0$ (note that the problem is invariant under a cyclic shift of indices (see [7] or a version of this paper in [5]) and so there is an equivalence class of special cases; in fact in [7] another representant is considered). By scaling (again, see [7] for details of this important aspect) one arrives at

$$
\begin{array}{r}
N_{0}(t+1)=\frac{1+\bar{I}}{1+I(t)} N_{2}(t) \\
N_{1}(t+1)=N_{0}(t) \\
N_{2}(t+1)=N_{1}(t)
\end{array}
$$

with

$$
I(t):=c_{0} N_{0}(t)+\left(1-c_{0}\right) N_{2}(t)
$$

parameterized by $c_{0}$ and the coexistence steady state value $\bar{I}$ of the scalar environmental condition $I$. Note that all density dependence is described by the function $h_{2}(I)$ which can be interpreted (if the census moment is just before the reproduction) as an expected number of offspring of an adult individual after a year, or written out in detail

$$
h_{2}(I)=E(I) p_{E}(I) p(I),
$$

where $E$ is the number of eggs per reproducing individual, $p_{E}$ is the probability for an egg to survive and to hatch and $p$ is the survival probability during the first year of life. Any of these quantities can depend on the environment as described by $I$. In the LPA model (see [4]) it is foremost $p_{E}$ that does depend on $I$ : the youngest (larvae) and the oldest (adults) age classes cannibalize on eggs. Of course, equation (6.1) is not an LPA model, it can be considered just as a simplified version of a particular case of it, because, firstly, there is no adult cannibalism on pupae, secondly, adults must die after reproduction and, thirdly, the density dependence is of Beverton-Holt (not Ricker) type. Notice also that a time unit in the LPA model is two weeks, not a year. However, we can interpret the model equation (6.1) as describing the dynamics of an (imaginary) semelparous species with three age classes from which the youngest and the oldest cannibalize on eggs and the middle one is not feeding.

For salmon we can suggest another mechanism that corresponds to the model equation (6.1) but with the productivity $E$ depending on $I$. Imagine that the negative effect of a high fry density is still noticeable the next year. Then $I$ has the form of equation (6.2), possibly with $c_{0}$ rather small.

Two shifted versions of equation (6.1) by the cyclic shift of indices could be very well interpreted for cicadas. In this case we choose the census moment to be just after reproduction. Let the first year survival $h_{0}$ be density dependent, with only the two young age classes entering competition $\left(c_{2}=0\right)$, then a possible underlying mechanism is competition for accommodation on grass roots while older larvae replaced themselves to tree roots (it is known that cicada larvae feed first on grass roots and later on tree roots). The second shifted version of equation (6.1) with the density dependence in $h_{1}$ and $c_{0}=0$ describes similar competition among older age classes on tree roots. 
Of the four Jury conditions that precisely guarantee the linearized stability of the coexistence steady state of equation (6.1) given by

$$
\bar{N}=\bar{I}\left(\begin{array}{l}
1 \\
1 \\
1
\end{array}\right),
$$

three are always satisfied. The remaining one reads

$$
1-2 c_{0}^{2}+\frac{1}{\bar{I}}\left(2-3 c_{0}\right)>0
$$

(cf. Lemma 11.3 and the calculations that follow it in [7]). This condition corresponds to a Neimark-Sacker bifurcation. If it fails, two complex conjugated eigenvalues of the steady state quit the unit circle. In general, an invariant circle appears as a result of this bifurcation. Numerical simulations for some values of $\bar{I}$ show that this is also the case for our system (see phase portraits below). For $c_{0} \leq 2 / 3$ equation (6.4) is satisfied but not for $c_{0}$ close to 1 . We define $\tilde{c}_{0}$ to be the unique zero of the left hand side.

When at the initial time $N_{1}$ and $N_{2}$ are both zero, equation (6.1) leads to the single year class (SYC) dynamics

$$
N_{0}(t+3)=\frac{1+\bar{I}}{1+\left(1-c_{0}\right) N_{0}(t)} N_{0}(t)
$$

with a fixed point

$$
\tilde{N}_{0}=\frac{1}{1-c_{0}} \bar{I}
$$

From the form of equation (6.5), in particular the monotonicity of the function defining the right hand side, it is clear that the fixed point is a global attractor for the SYC dynamics. But what about transversal stability? Can any of the two missing year classes invade successfully, given the 3-periodic environmental conditions corresponding to the SYC fixed point equation (6.6) of the full-life-cycle map?

The multipliers providing the answer are

$$
\begin{aligned}
& 1+\bar{I} \text { for year class " } 1 \text { " } \\
& \frac{1+\bar{I}}{1+\frac{c_{0}}{1-c_{0}}} \text { for year class " } 2 \text { ". }
\end{aligned}
$$

So year class "1" can always invade successfully (and the SYC fixed point is never transversally stable for the special case of equations (6.1) and (6.2)), while year class " 2 " can invade if $c_{0}<1 / 2$, but cannot if $c_{0}>1 / 2$.

The key point about equations (6.1) and (6.2) is that one can prove rather easily (see below) that for $c_{0}>1 / 2$ year class "1", once it has invaded successfully, actually outcompetes year class " 0 " so that the end result is that we are again in a SYC fixed point of the full-life-cycle map, but now with year class " 1 " ruling the world. The cyclic symmetry makes success illusive: next year class "2" can invade and take over, after which year class " 0 " finds itself in ideal conditions to regain power and so on and so forth. In more technical terms: the cyclic symmetry turns the connecting orbits between fixed points of the full-life-cycle map into a heteroclinic cycle. 
When at the initial time $N_{2}$ is zero, equation (6.1) leads to the partially decoupled system

$$
\begin{aligned}
& N_{0}(t+3)=\frac{1+\bar{I}}{1+c_{0} \frac{1+\bar{I}}{1+\left(1-c_{0}\right) N_{1}(t)} N_{1}(t)+\left(1-c_{0}\right) N_{0}(t)} N_{0}(t) \\
& N_{1}(t+3)=\frac{1+\bar{I}}{1+\left(1-c_{0}\right) N_{1}(t)} N_{1}(t) .
\end{aligned}
$$

It follows that $N_{1}(t)$ converges for $t \rightarrow \infty$ to

$$
\tilde{N}_{1}=\frac{1}{1-c_{0}} \bar{I} .
$$

Hence the limiting version of the first recursion in equation (6.8) reads

$$
N_{0}(t+3)=\frac{1+\bar{I}}{1+\frac{c_{0}}{1-c_{0}} \bar{I}+\left(1-c_{0}\right) N_{0}(t)} N_{0}(t) .
$$

If $c_{0}>1 / 2$ then $c_{0} /\left(1-c_{0}\right)>1$ and accordingly $N_{0}(t) \rightarrow 0$ for $t \rightarrow \infty$. If, on the other hand, $c_{0}<1 / 2$ then $N_{0}(t)$ converges for $t \rightarrow \infty$ to

$$
\tilde{\tilde{N}}_{0}=\frac{1-2 c_{0}}{\left(1-c_{0}\right)^{2}} \bar{I}
$$

and the three points

$$
\left(\begin{array}{c}
\tilde{\tilde{N}}_{0} \\
\tilde{N}_{1} \\
0
\end{array}\right),\left(\begin{array}{c}
0 \\
\tilde{\tilde{N}}_{0} \\
\tilde{N}_{1}
\end{array}\right),\left(\begin{array}{c}
\tilde{N}_{1} \\
0 \\
\tilde{\tilde{N}}_{0}
\end{array}\right)
$$

form a period 3 orbit with one year class missing. The multiplier determining the fate of the missing year class, when introduced in small numbers, equals

$$
\frac{1+\bar{I}}{1+c_{0} \tilde{N}_{0}}>1
$$

so we see that the situation with two year classes present and one missing is always unstable.

Now let's focus on $c_{0}>1 / 2$. The heteroclinic cycle can be either a repellor or an attractor. In [10, Chapter 17] it is explained, how one determines the stability of heteroclinic cycles for continuous time dynamical systems generated by differential equations. A key point is that one can give a criterion in terms of the local behaviour near the saddle points (where nearby orbits spend most of their "time"). When there is symmetry, like in our case, this behaviour is the same for all saddle points, so it suffices to look at only one of them. Translating the arguments of [Hofbauer \& Sigmund] to the present discrete time situation, one finds that the deciding quantity is the product

$$
\frac{(1+\bar{I})^{2}}{1+\frac{c_{0}}{1-c_{0}} \bar{I}}
$$

of the two multipliers in equation (6.7). For $c_{0}=\bar{c}_{0}$, with

$$
\bar{c}_{0}=\frac{\bar{I}+2}{\bar{I}+3}
$$


this product equals 1 . For $c_{0}<\bar{c}_{0}$ it exceeds 1 and the heteroclinic cycle is a repellor while for $c_{0}>\bar{c}_{0}$ it is less than 1 and the heteroclinic cycle is an attractor. Note that $\bar{c}_{0}>\tilde{c}_{0}$ (indeed, equation (6.4) is not satisfied for $c_{0}=\bar{c}_{0}$ ).

The symbolic pictures below summarize the information we have obtained. In [7] we describe simulations of a shifted version of the recursion equations (6.1) and (6.2) with constant $h_{1}$ and $h_{2}$ and all $c_{i} \geq 0$.
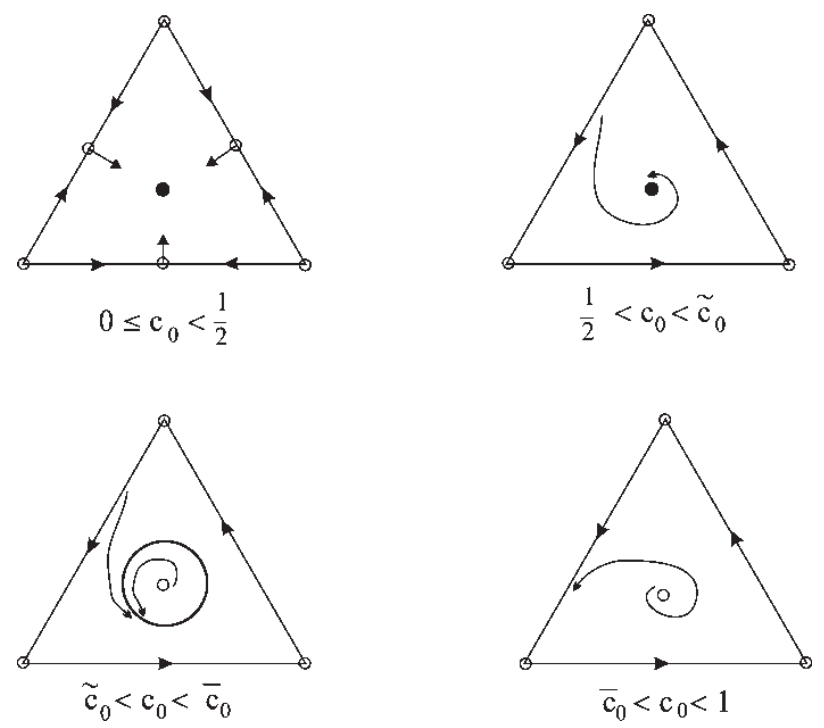

The numerical experiments for $c_{2}=0$ corroborate that sequence of phase portraits and (at least, for some values of $\bar{I}$ ) no other types of behaviour are found.

We see that we have coexistence of all year classes if the impact of the age class " 0 " (which does not suffer from competition) is relatively small or, using the LPA-like interpretation above, if adult cannibalism on eggs (that they produce themselves) is more pronounced than larvae cannibalism. If, on the contrary, it is larvae who eat eggs, we are likely to end up with competitive exclusion: it is better for a population not to have larvae and eggs simultaneously. However we never obtain a complete synchronization (SYC point is unstable): if we introduce several eggs during pupae-stage of the resident synchronized population, they will survive and this brood will overthrow the resident, because their larvae feed on resident's eggs.

The pictures above are to be called "symbolic" for two reasons

- they depict the dynamics on the attracting carrying simplex (which hasn't yet been shown to be a well-defined object);

- they provide a global extension of the local information that we deduced.

Extensive numerical testing confirmed the pictures, so we feel encouraged to conjecture that they are not just symbolic but that they provide an accurate description of the dynamics. It is an open problem to verify this conjecture or to disprove it. Jim, give it a try! 


\section{References}

[1] Bulmer, M.G., 1977, Periodical insects, The American Naturalist, 111, 1099-1117.

[2] Cushing, J.M., 2003, Cycle chains and the LPA model, Journal of Difference Equations and Applications, 9(7), $655-670$.

[3] Cushing, J.M., 1998, An introduction to structured population dynamics. CBMS-NSF Regional Conference Series in Applied Math, Vol. 71 (Philadelphia: SIAM).

[4] Cushing, J.M., et al. 2003, Chaos in Ecology (Academic Press, Elsevier Science).

[5] Davydova, N.V., 2004, Old and Young. Can they coexist? Ph.D. Thesis of Utrecht University, Netherlands.

[6] Davydova, N.V., Diekmann, O. and van Gils, S.A., 2003, Year class coexistence or competitive exclusion for strict biennials?, Journal of Mathematical Biology, 46, 95-131.

[7] Davydova, N.V., Diekmann, O., van Gils, S.A., On circulant population. I. The algebra of semelparity. Linear Algebra and its Applications, 398, 185-243.

[8] Diekmann, O., Gyllenberg, M., Huang, H., Kirkilionis, M., Metz, J.A.J. and Thieme, H.R., 2001, On the formulation and analysis of general deterministic structured population models. II. Nonlinear theory, Journal Mathematical Biology, 43, 157-189.

[9] Diekmann, O., Gyllenberg, M. and Metz, J.A.J., 2003, Steady state analysis of structured population models, Theoretical Population Biology, 63, 309-338.

[10] Hofbauer, J. and Sigmund, K., 1998, Evolutionary Games and Population Dynamics (Cambridge: Cambridge University Press).

[11] Smith, H.L., 1998, Planar competitive and cooperative difference equations, Journal of Difference Equations and Applications, 3, 335-357.

[12] Wang, Y. and Jiang, J., 2002, Uniqueness and attractivity of the carrying simplex for discrete-time competitive dynamical systems, Journal of Differential Equations, 186, 611-632. 